\title{
A novel approach to the island of stability of super-heavy elements search
}

\author{
A. Wieloch $^{1 *}$, M. AdamczyK ${ }^{1}$, M. Barbui $^{2}$, N. Blando $^{2}$, \\ G. Giuliani ${ }^{2}$, K. Hagel ${ }^{2}$, E-J. Kim${ }^{2}$, S. Kowalski ${ }^{3}$, Z. MajkA ${ }^{1}$, \\ J. Natowitz ${ }^{2}$, K. Pelczar ${ }^{1}$, R. Peaneta ${ }^{1}$, K. Schmidt ${ }^{3}, Z$ Z. $\operatorname{Sosin}^{1 \dagger}$, \\ S. Wuenschel ${ }^{2}$, K. Zelga ${ }^{1}$ and H. Zheng ${ }^{2}$
}

${ }^{1}$ M. Smoluchowski Institute of Physics, Jagiellonian University, Lojasiewicza 11, 30-348, Kraków, Poland

${ }^{2}$ Cyclotron Institute, Texas A\&M University, TX, USA

${ }^{3}$ University of Silesia, Katowice, Poland

\begin{abstract}
It is expected that the cross section for super-heavy nuclei production of $Z>118$ is dropping into the region of tens of femto barns. This creates a serious limitation for the complete fusion technique that is used so far. Moreover, the available combinations of the neutron to proton ratio of stable projectiles and targets are quite limited and it can be difficult to reach the island of stability of super heavy elements using complete fusion reactions with stable projectiles. In this context, a new experimental investigation of mechanisms other than complete fusion of heavy nuclei and a novel experimental technique are invented for our search of super- and hyper-nuclei. This contribution is focused on that technique.
\end{abstract}

\section{Introduction}

The history of super-heavy elements (SHEs) begins in the late 50-s. At that time the impressive progress in accelerator technology started and highly

*email: andrzej.wieloch@uj.edu.pl

${ }^{\dagger}$ Deceased 24 September 2015. 
efficient heavy ion sources were developed. New accelerators offered irradiation of target nuclei with high intensity beams of heavy ions which opened the door for the creation of SHEs [1]. In year 1957 G. Scharff-Goldhaber suggested a new region of existence of SHEs, the so called "island of stability", centered at nucleus ${ }_{126}^{310} X[2]$ and theoreticians W.D. Myers and W.J. Świtecki [3] calculated in 1966 that the center of the island should be located at $Z=126$ and neutron number $N=184$ while at the same time A. Sobiczewski, F.A. Gareev and B.N. Kalinkin [4] located this center at $Z=114$, $N=184$. Such a region of enhanced stability of nuclei, was possible solely due to the quantum effects. In contrast,a widely used liquid drop model was predicting disintegration of a nucleus already at $Z=104$.

Inspired by theory and new technological possibilities experimentalists initiated searches for SHEs and for the island of stability. To reach these goals a "cold" [5,6] and "hot" [7] complete fusion reactions in nuclear collisions were used where the excitation energies were $10-15 \mathrm{MeV}$ and $30-50$ $\mathrm{MeV}$, respectively. Unfortunately, the cross section for SHEs production in fusion reactions is decreasing rapidly by a factor of 4 with each $\mathrm{Z}$ reaching about $1 p b$ for element $Z=112$ [8]. This is a very serious limitation to the synthesis of the next elements. Moreover, half-lives of the heaviest nuclei were becoming very short, a few tens of $\mu s$. For even heavier nuclei one can expect that the cross section for their production is dropping into the region of tens of $f b$. Presently it is difficult to reach such low cross sections experimentally because of accessible intensities of ion beams. Radioactive ion beams (RIB) facilities are not a remedy as the intensities of neutron reach secondary beams delivered by RIB facilities are far too low.

So far we know fifteen SHEs starting from atomic number $Z=104$ and ending on $Z=118$. LBNL Berkely produced elements with $Z=104-106$ $[9,10]$, GSI Darmstadt created elements with $Z=107-112[5,11,12]$, JINR Dubna where elements with $Z=104-107,113-118$ [7,13-16] were produced and RIKEN Tokyo discovered element with $\mathrm{Z}=113$ [6].

However, the question How heavy can an atomic nucleus be? is still under debate and the existence of the stability island of SHEs is not yet experimentally confirmed.

\section{Alternative reaction mechanism for super- and hyper-heavy element production}

In order to overcome the constraints on SHEs production described in the introduction our groups began in 2002 to investigate massive transfer (multi 
nucleon transfer) reactions between heavy projectile (e.g. $A u$ ) and heavy fissile target nuclei (e.g. Th) [17].

More detailed discussion on the reaction scenario for the SHEs formation can be found in [18] and references quoted therein. Here we recall only the main points. If a large portion of mass is transferred from one reaction partner to the other, conditions similar to those of the fusion reactions might be met e.g. small angular momenta of the combined masses and a very heavy system can be formed. The majority of products originating from low-energy nuclear fission are neutron-rich nuclei. If such a fission fragment is transferred to the projectile nucleus as in the proposed collision scenario the fusion probability can be strongly enhanced due to the neutron excess. For more neutron rich heavy nuclei with proton/neutron numbers near the closed shells, then the survival probability of the formed SHE will increase as the fission barrier of the resulting SHE will be high. Besides, if the transfer process of the fission (or massive) fragment takes place at the peripheral collision some of the resulting systems may be formed with small excitation energy. In that case its survival probability again increases. Both factors may play a major role in an expected production of the SHEs and the fission or multinucleon transfer can be a kind of "ion source" delivering a wide spectrum of different heavy ions. Therefore, nature itself can choose the most appropriate fission fragment that will fuse with other reaction partner in order to produce a SHE. Using for example the projectile with $Z=79$ $(A u)$ and the target nucleus with $Z=90(T h)$ we may be able to explore production of SHEs and possibly hyper-heavy nuclear systems in the regions of atomic numbers $Z \approx 114$ and $Z \approx 126$ where the shell effects might play an important rule $[19,20]$.Even higher regions of $Z$ can be explored with such a technique.

However, one should remember that in the proposed reactions a relatively long interaction time will be involved. As a result, quite high excitation energy of the reaction partners can be generated and in those cases the survival probability of the produced SHE nucleus can decrease dramatically. The survival will depend strongly on the excitation energy distribution.

\section{$3 \quad$ Experimental setup}

One can expect that in the proposed reaction mechanism SHEs will be produced with a wide spectrum of atomic numbers and velocities and have relatively broad angular distributions. Thus the "classical" Wien filters applied for a complete fusion SHEs production where $Z$ and velocity of syn- 
thesized SHEs are well defined can not be utilized. Instead, a completely new experimental setup was introduced in research presented here.

Our experiments started in 2002 at K500 accelarator of Cyclotron Institute of TAMU using an efficient magnetic velocity filter, the superconducting solenoid (BigSol) [17]. We investigated reaction ${ }^{197} \mathrm{Au}(7.5 \mathrm{~A} . \mathrm{MeV})+{ }^{232} \mathrm{Th}$. Obtained results showed [21] that nuclei with $Z$ above 100 can be produced via heavy mass transfer described in the former chapter.

In 2012, a new detection system was introduced into our SHE search [18]. The active catcher (AC) construction was based on an idea to identify production of SHEs via characteristic alpha decay. Indeed, many SHEs are expected to decay by alpha emission with unusually high energies [22] and this delivers a method to distinguish such decays from that of lighter elements. A prototype of the AC detection system was used for the first time in August 2013 in a test experiment. A final construction/geometry of the AC detection system (see Fig. 1) was tested in measurements in the summer of 2014.

\section{Schematics of detection setup}

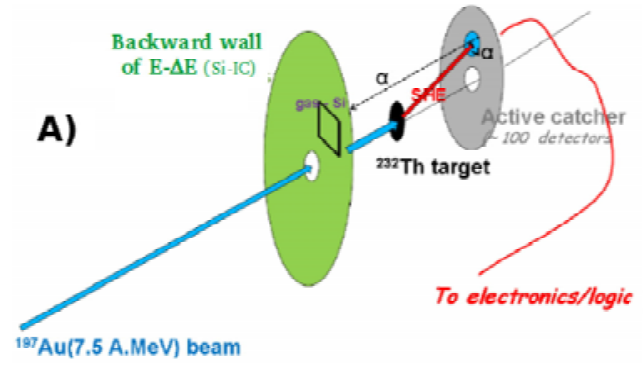

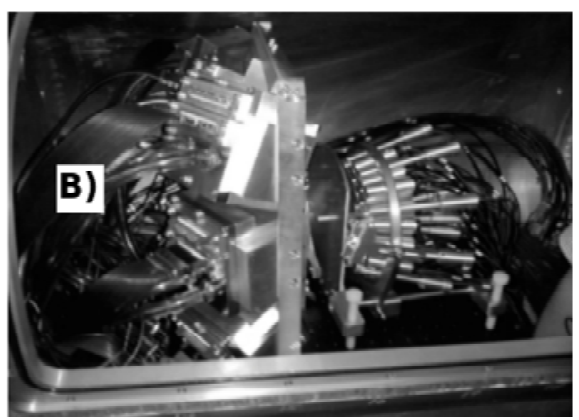

Figure 1: A) Schematics drawing of the AC detection system. B) Photo of the constructed AC system mounted inside the vacuum chamber at TAMU (on the left $\Delta E-E$ telescopes and on right the $\mathrm{AC})$.

In our experimental setup the detection and identification of SHEs is a two stage process. In the first step the heavy reaction products are implanted and registered in the $\mathrm{AC}$ units which are located downstream from the target. In the second stage the alpha particles emitted are detected either by the AC detection unit in which the heavy reaction product was implanted and decayed and, for those emerging from the AC in the backward hemisphere, by an array of $\Delta E$ (gas) $-E(\mathrm{Si})$ telescopes. and/or Spontaneous fission decays of implanted heavy nuclei can be recognized by the AC, also.

Each unit of the AC detector consists of a fast plastic scintillator (BC418) where the reaction products are implanted, an aluminum cylinder which 
serves as a light guide and the photomultiplier tube (PMT R9880U-110) with an active window diameter of $\phi=8 \mathrm{~mm}$ (for a schematic view see [18]). To get optimal granularity of the AC detector system two sets of aluminum cylinders were applied (see Fig.1B). At the angles closest to the beam 18 units are mounted $\phi=8 \mathrm{~mm}$ diameter (long cylinders) and at angles larger than $15^{\circ} 36$ units are used with diameter equal $19 \mathrm{~mm}$ (short cylinders). For a better covering of the available space at the largest angles 9 units with long cylinders are added. The whole detection setup is quite compact, units of the $\mathrm{AC}$ system and $\Delta E-E$ telescopes of the backward wall are located less then $10 \mathrm{~cm}$ from the target. Such a configuration gives capability of measuring decays of very short lived SHEs of the order of $10 \mathrm{~ns}$.

The other advantages of the AC detection system are a very good time resolution (the scintillation pulse width is about $5 \mathrm{~ns}$ with rise time $1 \mathrm{~ns}$ ) and a dynamical range which allows to distinguish between deposited heavy reaction products and decayed alpha particles.

A high background is a natural consequence of the approach we are using for SHE search. In order to eliminate unwanted events such as scattered beam, projectile like fragments and a few nucleon transfer reaction products from data acquisition (ACQ) a dedicated trigger of an ACQ was built. The first block of electronics shown in Fig. 2A produces logical signals from analog pulses of the PMT modules of the AC detector. A final formation of the trigger is done by two FPGA boards (see Fig.3).

A very important role in the trigger formation is played by the electronics which converts the RF cyclotron signal to a logical pulse in the way that one logical level (in our case it is logical "0") has a duration of the beam burst from the cyclotron which is 5 ns at the target and level logical "1" of $50 \mathrm{~ns}$ width equal to a time period with no beam particles at the target (see Fig.2B).

The FPGA board shown in Fig.3 produces logical $O R$ of all of the PMT pulses. Then the logical $A N D$ vetoes logical $O R$ during the time period when the beam particles hit the target $(5 \mathrm{~ns}$ ). The logical $O R$ (marked in the Fig. 3 as IC-Si trigger) allows generation of the ACQ trigger when the alpha particle is detected by a $\Delta E-E$ backward detector. This final trigger activates the ACQ system that is composed of two 32 Channel fast Flash ADCs V1742. The pulse shapes of the PMT modules are recorded by Flash ADCs while signals from $\Delta E-E$ detectors are picked up by another independent acquisition. To assure event agreement for both ACQs an event counter is followed and marked as "EvtCount" in Fig. 3. Module V1742 was working with sampling rate of $1 \mathrm{Gs} / \mathrm{s}$ while the width of the common time window for the detection systems was $1 \mu$. Location of the ACQ trigger 
in V1742 time window enables to record interesting event time structure backwards in time upto $1 \mu s$.

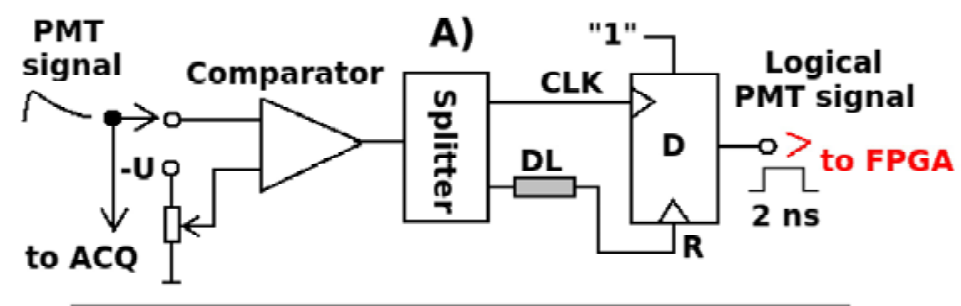

B)

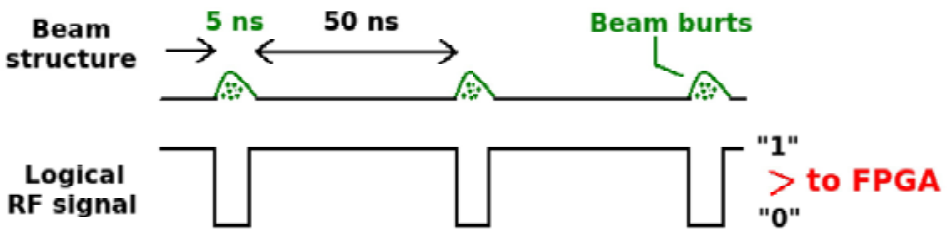

Figure 2: A) PMT spectroscopic signal from each module of the AC is converted by dedicated electronics to the fast logical signal. B) Logical RF pulses produced from the sinus shape of the original RF signal are converted in a such way that the logical " 0 " fits to the beam burst duration. Both types of signals are delivered to the trigger production logic build on the FPGA module.

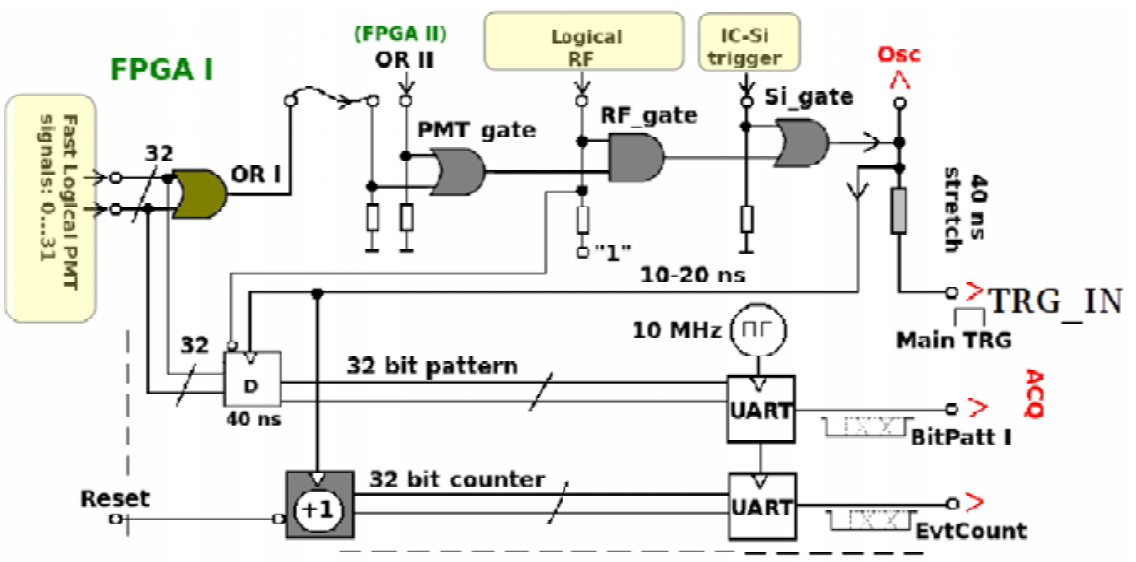

Figure 3: Logic constructed on FPGA boards served to produce main trigger. Schematics of only one FPGA is shown while the other has the same logic. 
Fig.4 presents example of two interesting events extracted from measurement in summer 2014. In both cases acquisition was triggered by an $\alpha$ particle detected in one of $\Delta E-E$ detectors. With such a trigger flash ADC recorded pulse shapes generated by the PMTs of the AC units number 11 (panel A) and 24 (panel B). For the first event the distance between peaks of the pulses is $(113-22)$ ns while for the second event this distance is $(275-133) n s$. If these time differences are divided by the characteristic time of beam bunches equal to $55 \mathrm{~ns}$ we obtain 1.65 and 2.58 values. These two numbers prove that in both cases at least one of the detected particles came between beam bursts. These examples clearly show that the AC detection system is able to register $\alpha$ decays of very short lived isotopes.
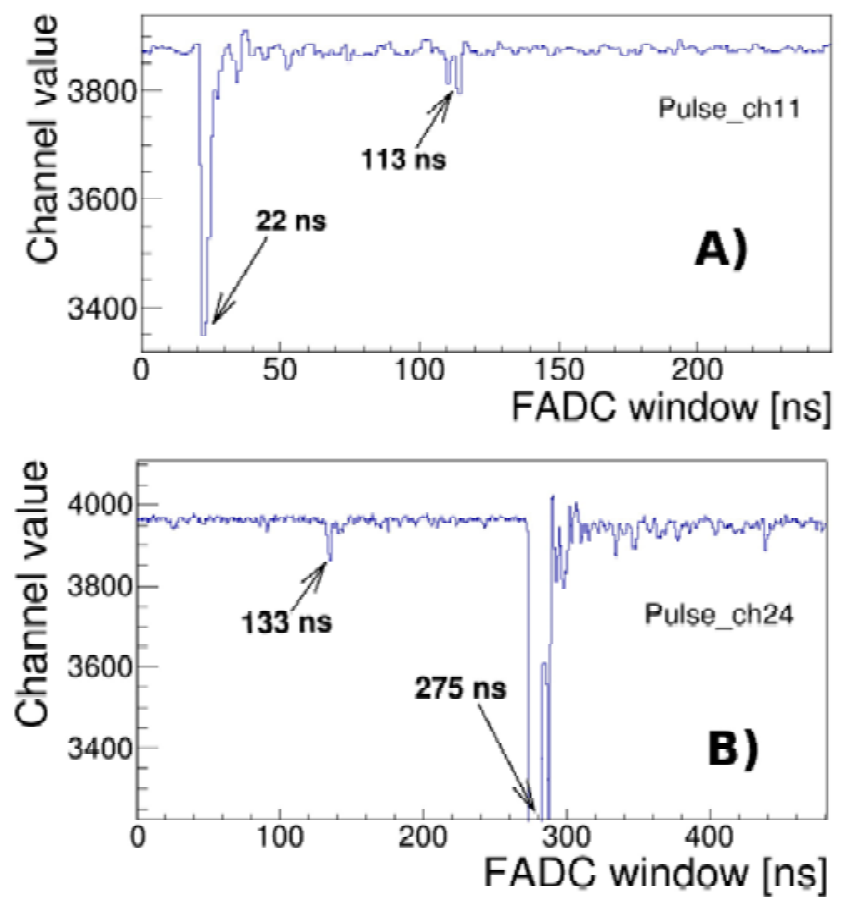

Figure 4: Flash ADC recorded pulses for two events A) and B) while ACQ system was triggered by signal of $\alpha$ particle detected in of $\Delta E-E$ detector. A) Energy of $\alpha$ particle was $9.90 \mathrm{MeV}$ while in case of event B) $\alpha$ particle has energy $7.60 \mathrm{MeV}$.

Presently further improvements in the active catcher detection system are underway. Especially, to get better quality pulses from the scintillator of the $\mathrm{AC}$ units we installed lucite light guides with the shapes optimized 
for a short and long aluminum cylinders. Additionally computer driven thresholds of the first electronic block are being implemented. The next measurements are scheduled in early November of 2015.

\section{Acknowledgments}

This work is supported by the National Science Center in Poland, contract no. UMO-2012/04/A/ST2/00082, by the U.S. Department of Energy under Grant No. DE-FG03-93ER40773 and by the Robert A. Welch Foundation under Grant A0330.

\section{References}

[1] See e.g. Kumar K., in Super Heavy Elements, edited by Adam Hilger, (Bristol and New York) 1989.

[2] Scharff-Goldhaber G., Nucleonics, 15 (1957) 122.

[3] Myers W.D., Świtecki W.J., Nucl. Phys., 81 (1966) 1.

[4] Sobiczewski A. et al., Phys. Lett., 22 (1966) 500.

[5] Hofmann S. and Münzenberg G., Rev. Mod. Phys., 75 (2000) 733.

[6] Morita K. et al., J. Phys. Soc. Jpn., 73 (2004) 2593.

[7] Oganessian Yu., Phys. Rev., C74 (2006) 044602.

[8] Hofmann S. et al., Eur. Phys. J., A14 (2002) 147.

[9] Ghiorso A. et al., Phys. Rev. Lett., 22 (1969) 1317.

[10] Ghiorso A. et al., Phys. Rev. Lett., 33 (1974) 1490.

[11] Hofmann H. et al., Nucl. Phys., A496 (1989) 269.

[12] Hofmann S. et al., Z. Phys., A350 (1995) 281.

[13] Oganessian Yu. et al.,Nucl. Phys., A273 (1976) 505.

[14] Oganessian Yu., Phys. Rev., C63 (2001) 011301R.

[15] Oganessian Yu., J. Nucl. Radiochem. Sci., 3 (2002) 5. 
[16] Oganessian Yu., LNL Report, Berkeley, UCRL-ID-151619 (2003).

[17] Materna T. et al., Progress in Research April 1, 2003-March 31, 2004, Cyclotron Institute, Texas A\&M University, 2004 pp. II-17.

[18] Majka Z. et al., Acta Phys. Pol., B45 No 2 (2014) 279.

[19] Zagrebaev V.I. et al. J. Phys.: Conference Series, 420 (2013) 012001.

[20] Zagrebaev V.I. and Greiner W., Phys. Rev., C87 (2013) 034608.

[21] Barbui M. et al., Int. J. Mod. Phys, E18 (2009) 1036.

[22] Bender M. and Heenen P.-H., J. Phys.: Conference Series, 420 (2013) 012002. 